\title{
Preparation and characterization of a novel silica aerogel as adsorbent for toxic organic compounds
}

\author{
Huijuan Liu ${ }^{\mathrm{a}, *}$, Wei Sha ${ }^{\mathrm{a}}$, Adrienne T. Cooper $^{\mathrm{b}}$, Maohong Fan ${ }^{\mathrm{c}}$ \\ a SKLEAC, Research Center for Eco-Environmental Sciences, Chinese Academy of Sciences, Beijing 100085, China \\ b Civil and Environmental Engineering, Temple University, Philadelphia, PA 19122, USA \\ ' School of Civil and Environmental Engineering, Georgia Institute of Technology, Atlanta, GA 30332, USA
}

\section{A R T I C L E I N F O}

\section{Article history:}

Received 29 June 2008

Received in revised form 6 November 2008

Accepted 19 November 2008

Available online 27 November 2008

\section{Keywords:}

Silica aerogel

Ambient drying

Adsorbent

Toxic organic compounds

\begin{abstract}
A B S T R A C T
Hydrophobic and hydrophilic silica aerogels were prepared from cheap waterglass precursors via surface modification of wet gels, ambient pressure drying (APD) route and calcination treatment. Xerogel was also synthesized as a comparison to test the influence of microstructure on the adsorption behaviours. Ethanol (EtOH)/chlorotrimethylsilane (TMCS)/hexane solution was employed to replace the surface silanol groups $(\mathrm{Si}-\mathrm{OH})$ with alkyl, and to prevent the condensation and shrinkage of the gel structure during the APD process. The synthesized silica aerogels, characterized by using Fourier transform infrared spectra (FTIR), nitrogen adsorption/desorption measurement, element analyses and differential scanning calorimeter (DSC), etc., are porous and translucent solids with surface area of $546-907 \mathrm{~m}^{2} / \mathrm{g}$. The transition temperature from hydrophobic to hydrophilic was tested around $395^{\circ} \mathrm{C}$, which corresponds to the disappearance temperature of the $-\mathrm{CH}_{3}$ peaks in FTIR. The materials were tested for adsorption capacities on rhodamine $\mathrm{B}(\mathrm{RhB})$ and dieldrin, which represented soluble and slightly soluble organic compounds, respectively. Results showed that the hydrophobic silica aerogel exhibited strong adsorption capacity on slightly soluble organic compounds while hydrophilic silica aerogel was much more effective on adsorbing soluble compounds from aqueous solution. Adsorption properties of the silica aerogel remain stable after 5 adsorption/desorption cycles.
\end{abstract}

(c) 2008 Elsevier B.V. All rights reserved.

\section{Introduction}

Widespread environmental problems are an urgent global issue requiring immediate attention. The evaluation of complex industrial societies has led to the creation and proliferation of a vast variety of contaminants from persistent organic pollutants (POPs) to oil-spills $[1,2]$. A common approach to removal of toxic organic pollutants from water is the adsorption process using porous materials. Granular activated carbon (GAC) is a widely used adsorbent due to its high adsorption capacity and availability [3]. However, GACs display disadvantages such as low selectivity and limited modification flexibility. Thus, a well-designed alternative adsorbent with a surface property that is easily modified is needed to overcome the shortcomings associated with GAC.

Traditionally, adsorbents are porous materials with high specific surface areas, large pore volumes, well-developed pore distributions, and special pore surface chemistries. Silica aerogel, a unique material with wide application [4-6], has been increas-

\footnotetext{
* Corresponding author. Tel.: +86 10 62849160; fax: +861062849160. E-mail addresses: hjliu@rcees.ac.cn (H. Liu), hewsay@gmail.com (W. Sha), acooper@temple.edu (A.T. Cooper), mfan3@mail.gatech.edu (M. Fan).
}

ingly researched as an adsorbent due to its high porosity (up to 99\%) and ease of surface modification. It shows high adsorption efficiencies for many different compounds in water [7-9]. Traditional preparation methods of silica aerogel involve the process of supercritical drying, which can avoid capillary stress and associated drying shrinkage. However, the supercritical drying process is energy intensive and can be dangerous, which leads to high costs and results in limited practical applications [10,11].

Recently, a new ambient pressure drying (APD) technique has been developed to overcome the disadvantages of conventional supercritical aerogel process, and made the manufacture and application of silica aerogel in large scale possible [12-15]. The key steps of this novel preparation method were solvent exchange and surface modification before the APD treatment. Schwertferger et al. [15] developed a process in which one-step solvent exchange and surface modification were simultaneously progressed by using a HMDSO (hexamethyldisiloxane)/TMCS (trimethylchlorosilane) solution for modification of the wet gels. Recently, Shi et al. [14] also synthesized silica aerogels via one-step solvent exchange/surface modification of wet gels using an ethanol/TMCS/heptane solution.

In this study, hydrophobic silica aerogel was prepared from cheap waterglass precursors using EtOH/TMCS/hexane solution for modification of wet gels via the APD route. The synthesized 
hydrophobic aerogel product was calcined at $500{ }^{\circ} \mathrm{C}$ for $30 \mathrm{~min}$ to further modify its surface chemistry. Rhodamine $\mathrm{B}(\mathrm{RhB})$ and dieldrin, representing soluble and slightly soluble organic compounds, respectively, were chosen to examine their adsorption on different types of the prepared adsorbate.

\section{Experimental}

\subsection{Materials}

Waterglass $\left(\mathrm{Be}^{\mathrm{O}}=40, \mathrm{Na}_{2} \mathrm{O}: \mathrm{SiO}_{2}\right.$ molar ratio $\left.=1: 3.3\right)$ and $\mathrm{NaOH}$ (A.R.) was purchased from Beijing Chemical Works (Beijing China). Ethanol (A.R.) was obtained from Beijing Chemical Reagent Company (Beijing China). n-Hexane (HPLC) was ordered from Fisher Scientific Company (USA), and TMCS (A.R.) was gotten from Sinopharm Chemical Reagent Co., Ltd. (Shanghai, China). The targeted analytes RhB (A.R.) and dieldrin were acquired from Beijing Chemical Works and Environmental Protection Center of the Chinese Department of Agriculture, respectively.

\subsection{Preparation of absorbents}

\subsubsection{Preparation of silica wet gels and Xerogel $C$}

Silica wet gels were prepared with waterglass as starting materials. The waterglass was diluted with deionized water (waterglass/deionized water $(\mathrm{v} / \mathrm{v})=1: 4)$. The diluted solution went through an ion exchange column filled with strongly acidic type cation exchange resin to remove $\mathrm{Na}^{+}$. The $\mathrm{pH}$ of the collected silica sol was in the range of $2-3$. Then $1.0 \mathrm{~mol} / \mathrm{L} \mathrm{NaOH}$ solution was added to the silica sol to raise its $\mathrm{pH}$ to 5 for gelation. The obtained silica sols were stirred for $1 \mathrm{~min}$, and then transferred into a plastic beaker where the sols aged into hydrogels within about $15 \mathrm{~min}$. The next step was to immerse the hydrogels into deionized water and age them for $24 \mathrm{~h}$ at room temperature to strengthen the networks of the gels. Finally, the aged wet gels were dried at $60^{\circ} \mathrm{C}$, $80^{\circ} \mathrm{C}, 120^{\circ} \mathrm{C}$ and $180^{\circ} \mathrm{C}$ for $2 \mathrm{~h}$ separately in an oven, and the dried material was referred to as Xerogel $\mathrm{C}$.

\subsubsection{Solvent exchange/surface modification and preparation of Aerogel $A$ and $B$}

After gelation, the hydrogels were immersed into $50 \% \mathrm{H}_{2} \mathrm{O} / \mathrm{EtOH}$ solution and aged for $24 \mathrm{~h}$ at room temperature in order to further strengthen their networks. Then, EtOH/TMCS/Hexane mix solution was added to the strengthened wet gels to modify their surface chemistry property. After the preliminary experiments, the optimal molar ratio of pore water/TMCS/EtOH/hexane for modification was adopted as $2.5 / 0.8 / 1 / 1$. With the completion of the reaction between the wet gel and the mixed solution and through extrusion of pore water, the four modified gel samples were then separately dried at $60^{\circ} \mathrm{C}, 80^{\circ} \mathrm{C}, 120^{\circ} \mathrm{C}$ and $180^{\circ} \mathrm{C}$ for $2 \mathrm{~h}$. The dried aerogels were named Aerogel A. To further modify the property of the material, Aerogel A was calcined at $500^{\circ} \mathrm{C}$ for $30 \mathrm{~min}$ in an oven, and the products resulting from the process was called Aerogel B.

\subsection{Characterization}

The microstructure and morphology of the materials were observed with a HITACHI S-450 scanning electron microscope (SEM). Nitrogen adsorption/desorption isotherms of the materials were determined by an ASAP 2000 surface analyzer (Micromeritics Co., USA). Each run was performed on approximately $0.1 \mathrm{~g}$ of sample which was pretreated at $200^{\circ} \mathrm{C}$ under vacuum. The contact angle of Aerogel A was measured by photographing a water droplet on the aerogel surface. The thermal behavior of the dried materials was examined using differential thermal analysis (TG/SDTA 851). An elemental analyzer (PERKIN ELMER CE440) was used to study the elemental composition of the samples. FT-IR-Spectrometer Nicolet 5700 was employed to determine the vibration frequency in the functional groups of the materials.

\subsection{Adsorption capacity determination}

Two different organic compounds selected to test the adsorption effectiveness of the novel adsorbents with different hydrophobicity were $\mathrm{RhB}$ and dieldrin, representing soluble and slightly soluble organic compounds, respectively.

\subsubsection{Adsorption kinetics}

To investigate the adsorption effectiveness of the novel adsorbents on soluble RhB, $0.5 \mathrm{~g}$ Aerogel A, B and Xerogel C was added, respectively, into three $100 \mathrm{~mL}$ solutions containing $10 \mathrm{mg} / \mathrm{L} \mathrm{RhB}$. For the investigation of slightly soluble dieldrin, $0.1 \mathrm{~g}$ Aerogel A, B, and Xerogel $C$ was added, respectively, into three $150 \mathrm{~mL}$ solution containing $150 \mu \mathrm{g} / \mathrm{L}$ dieldrin. Batch adsorption experiments were carried out with agitation provided by a shaker at 170 revolutions per minute (rpm). The adsorption temperature was controlled at $25^{\circ} \mathrm{C}$ with an air bath. Samples were withdrawn periodically for analyses.

\subsubsection{Adsorption equilibrium}

Preliminary kinetic tests indicated that the adsorption equilibria of $\mathrm{RhB}$ and dieldrin were reached after $48 \mathrm{~h}$ because no further changes in adsorbate concentrations were observed.

$\mathrm{RhB}$ adsorption isotherms were obtained by mixing $0.5 \mathrm{~g}$ Aerogel B with $100 \mathrm{~mL}$ RhB solution of different initial concentrations; dieldrin adsorption isotherms were obtained by mixing $0.025 \mathrm{~g}$ Aerogel A with $150 \mathrm{~mL}$ dieldrin solution with different initial concentrations. The suspensions were shaken at $170 \mathrm{rpm}$, and the adsorption temperature was kept at $25^{\circ} \mathrm{C}$. Samples for analysis were taken after $48 \mathrm{~h}$ contact time.

\subsubsection{Reusability of aerogel adsorbents}

Multiple cycles of adsorption and desorption of RhB on Aerogel B and dieldrin on Aerogel A were performed to test the reusability of the prepared aerogel adsorbents. During the adsorption/desorption experiments, the adsorption steps were performed after complete desorption (regeneration) using thermal treatment. The regeneration of Aerogel A and B were achieved after removing adsorbed adsorbates and were dried at $300^{\circ} \mathrm{C}$ and $500^{\circ} \mathrm{C}$, respectively.

\subsection{Analytical procedures}

An UV/VIS spectrophotometer HITACHI U-3010 was employed to analyze the residual adsorbate of RhB in tested solutions at the absorption wavelength of $554 \mathrm{~nm}$. Dieldrin presented in water samples with trace levels, thus liquid-liquid extraction with n-hexane (GC grade, Fisher Scientific Company, USA) was conducted for its measurement. The analyses of residual concentrations of dieldrin were performed on an Agilent 6890N gas chromatograph (Agilent Technologies, USA), equipped with a split-splitless injector and a ${ }^{63} \mathrm{Ni} \mu$-electron capture detector. A HP-5 capillary column $(30 \mathrm{~m} \times 0.32 \mathrm{~mm}$ i.d., film thickness of $0.17 \mu \mathrm{m})$ was purchased from J\&W Scientific (USA). The temperature of column temperature was programmable and its initial value was set at $85^{\circ} \mathrm{C}$, then increased to $180^{\circ} \mathrm{C}$ at a rate of $10^{\circ} \mathrm{C} / \mathrm{min}$, held for $15 \mathrm{~min}$, and finally ramped to $280^{\circ} \mathrm{C}$ at a rate of $20^{\circ} \mathrm{C} / \mathrm{min}$ and held for $30 \mathrm{~min}$. The injector temperature was set at $250^{\circ} \mathrm{C}$ and the detector at $300^{\circ} \mathrm{C}$. High-purity ( $>99.999 \%$ ) nitrogen gas was used as the carrier gas with a flow rate of $1 \mathrm{~mL} / \mathrm{min}$ and a pressure of $16 \mathrm{psi}$. The $1 \mu \mathrm{L}$ sample was injected in splitless mode. Chromatograms were recorded and analyzed using the HP Chem-Station software. 


\section{Results and discussion}

\subsection{Mechanism of solvent exchange/surface modification}

In order to obtain high porous aerogel structure, elimination of capillary stress during drying is very important. In this study, the elimination of capillary stress was performed by pore water solvent exchange and surface modification of wet gel before ambient drying. EtOH/TMCS/hexane solution was used to replace the surface silanol groups $(\mathrm{Si}-\mathrm{OH})$ with alkyl, and to prevent the condensation and shrinkage of the gel structure during the APD process. When adding ethanol, hexane and TMCS to the wet gel, reactions occurred between the wet gel and EtOH/TMCS/Hexane solution. During the reaction of EtOH/TMCS/hexane solution with wet gel, a similar phenomena as illustrated in literature [15] can be observed: transparent yellow liquid (the aqueous $\mathrm{HCl}$ phase) coming out from the wet gel and staying in the hexane phase, while the resulting modified wet gels floated to the top of the derived solution of aqueous $\mathrm{HCl}$ phase mainly consisting of water extruded from the pores of wet gels and the formed $\mathrm{HCl}$. The major reactions involved with surface modification were as follows [14]:

$$
\begin{aligned}
& 2\left(\mathrm{CH}_{3}\right)_{3}-\mathrm{Si}-\mathrm{Cl}+\mathrm{H}_{2} \mathrm{O} \text { (pore water) } \\
& \quad \rightarrow\left(\mathrm{CH}_{3}\right)_{3}-\mathrm{Si}-\mathrm{O}-\mathrm{Si}-\left(\mathrm{CH}_{3}\right)_{3}+2 \mathrm{HCl} \\
& \left(\mathrm{CH}_{3}\right)_{3}-\mathrm{Si}-\mathrm{Cl}+\mathrm{CH}_{3} \mathrm{CH}_{2} \mathrm{OH} \rightarrow\left(\mathrm{CH}_{3}\right)_{3}-\mathrm{Si}-\mathrm{O}-\mathrm{CH}_{2} \mathrm{CH}_{3}+\mathrm{HCl} \\
& 2\left(\mathrm{CH}_{3}\right)_{3}-\mathrm{Si}-\mathrm{O}-\mathrm{CH}_{2} \mathrm{CH}_{3}+\mathrm{H}_{2} \mathrm{O} \text { (pore water) } \\
& \quad \rightarrow\left(\mathrm{CH}_{3}\right)_{3}-\mathrm{Si}-\mathrm{O}-\mathrm{Si}-\left(\mathrm{CH}_{3}\right)_{3}+2 \mathrm{CH}_{3} \mathrm{CH}_{2} \mathrm{OH} \\
& \left(\mathrm{CH}_{3}\right)_{3}-\mathrm{Si}-\mathrm{O}-\mathrm{Si}-\left(\mathrm{CH}_{3}\right)_{3}+2 \mathrm{HCl} \rightarrow 2\left(\mathrm{CH}_{3}\right)_{3}-\mathrm{Si}-\mathrm{Cl}+\mathrm{H}_{2} \mathrm{O} \\
& \left(\mathrm{CH}_{3}\right)_{3}-\mathrm{Si}-\mathrm{O}-\mathrm{CH}_{2} \mathrm{CH}_{3}+\mathrm{HCl} \rightarrow\left(\mathrm{CH}_{3}\right)_{3}-\mathrm{Si}-\mathrm{Cl}+\mathrm{CH}_{3} \mathrm{CH}_{2} \mathrm{OH} \\
& \left(\mathrm{CH}_{3}\right)_{3}-\mathrm{Si}-\mathrm{Cl}+\equiv \mathrm{Si}-\mathrm{OH} \rightarrow \equiv \mathrm{Si}-\mathrm{O}-\left(\mathrm{CH}_{3}\right)_{3}+\mathrm{HCl} \\
& \left(\mathrm{CH}_{3}\right)_{3}-\mathrm{Si}-\mathrm{O}-\mathrm{CH}_{2} \mathrm{CH}_{3}+\equiv \mathrm{Si}-\mathrm{OH} \rightarrow \equiv \mathrm{Si}-\mathrm{O}-\left(\mathrm{CH}_{3}\right)_{3}+\mathrm{CH}_{3} \mathrm{CH}_{2} \mathrm{OH}
\end{aligned}
$$

It is clear that TMCS react with ethanol, pore water and $\mathrm{Si}-\mathrm{OH}$ group in the wet gel. Because the reaction between the TMCS and pore water in the wet gel was so rapid, it would easily cause the gel to crack. Thus, during the process of solvent exchange/surface modification, controlling the reaction rate of the TMCS with the pore water is very important. Practically, the reaction between TMCS and ethanol decreases the reaction rate of TMCS with pore water, which is a favorable condition for obtaining crack-free aerogels. The molar ratio of EtOH/TMCS/hexane is a key factor in determining the properties of the silica aerogel. If the operation of adding ethanol, hexane solvent and TMCS modification reagent is appropriate, then monolithic silica aerogels with low density could be obtained. From the preliminary experiments, the optimum molar

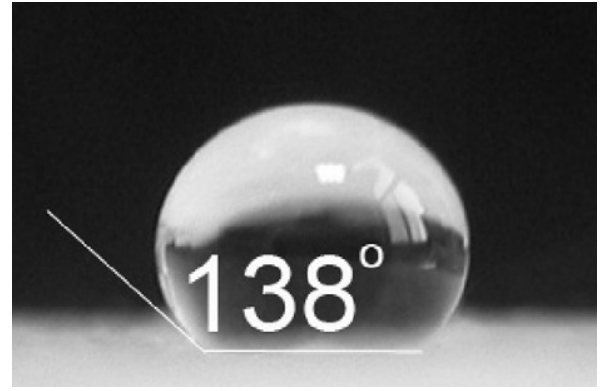

Fig. 2. Contact angle of Aerogel A.

ratio of pore water/TMCS/EtOH/hexane was 2.5/0.8/1/1 for silica surface modification [16]. This modification mechanism has been applied by Schwerfeger's et al. [15] and Shi et al. [14]. After the modification process, the hydrophilic property of the internal surface was modified to hydrophobic by isopropoxytrimethylsilane and TMCS that react with the $-\mathrm{OH}$ of the internal surface.

\subsection{Properties of silica aerogels}

\subsubsection{Microstructure of silica aerogels and xerogel}

Fig. 1 shows the SEM morphology of the three materials. Aerogel A, which was modified with surface methyl groups, exhibits a porous network structure. The aerogel skeleton of Aerogel B became thinner as the methyl groups on the pore surface were oxidized during calcination. After the serious volume shrinkage during the APD, the unmodified Xerogel $C$ did not show any porous microstructure under SEM observation, which reflects the importance of surface modification and elimination of capillary stress to obtain the porous aerogel structure [11,13].

\subsubsection{Hydrophobicity of aerogels and xerogel}

Fig. 2 shows a water droplet on the surface of monolith Aerogel A, which obviously had a strong hydrophobic character with a contact angle of $138^{\circ}$ due to the modified surface methyl group [10,16]. The surface modification was confirmed by FTIR and elemental analysis [16]. Aerogel B showed strong hydrophilicity and absorbed the water droplets immediately after contact as most of the surface methyl groups were oxidized during the calcination treatment at $500^{\circ} \mathrm{C}$. Xerogel C, which was not subjected to surface modification, was also hydrophilic. Its hydrophilicity was mainly due to presence of the hydrophilic group of $\mathrm{Si}-\mathrm{OH}$ on the surface.

\subsubsection{Textural properties of silica aerogels and xerogel}

Nitrogen adsorption/desorption method was used to analyze the textural properties of silica aerogels and xerogel. The specific surface area was determined using Brunauer-Emmett-Teller (BET) method. Pore size distribution and total pore volume of the materials were evaluated from the adsorption branch of nitrogen

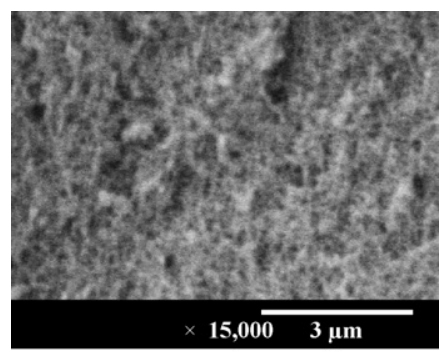

Aerogel A

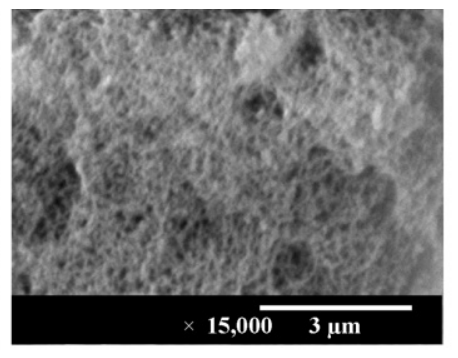

Aerogel B

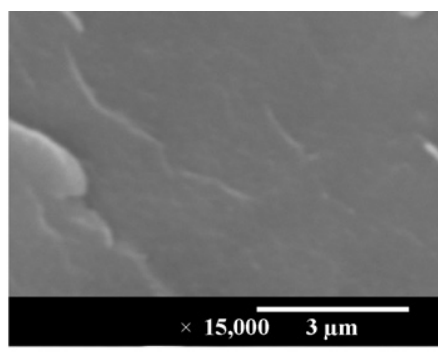

Xerogel C

Fig. 1. SEM pictures of Aerogel A, B and Xerogel C $(\times 15,000)$. 
Table 1

Textural properties of Aerogel A, B and Xerogel C.

\begin{tabular}{|c|c|c|c|c|c|}
\hline Sample & BET surface area $\left(\mathrm{m}^{2} / \mathrm{g}\right)$ & Average pore diameter (nm) & Pore volume $\left(\mathrm{m}^{3} / \mathrm{g}\right)$ & Carbon content & Hydrogen content \\
\hline Aerogel A & 546 & 17.6 & 2.40 & $12.91 \%$ & $2.97 \%$ \\
\hline Aerogel B & 907 & 21.0 & 2.95 & $0.89 \%$ & $0.23 \%$ \\
\hline Xerogel C & 788 & 3.2 & 0.64 & $1.03 \%$ & $1.01 \%$ \\
\hline
\end{tabular}

isotherms by using the Barrett-Joyner-Halenda (BJH) method. The experimental results on the specific surface areas, total pore volume and average pore diameter of the aerogel powders have been compiled in Table 1 along with the results of elemental analysis. Aerogel A was a porous material with high surface area $\left(546 \mathrm{~m}^{2} / \mathrm{g}\right)$, average pore size $(17.6 \mathrm{~nm})$ and total pore volume $\left(2.40 \mathrm{~m}^{3} / \mathrm{g}\right)$. The carbon and hydrogen contents of Aerogel A were $12.91 \%$ and $2.97 \%$, respectively, which were much higher than that of the other two materials. The calcination treatment at $500^{\circ} \mathrm{C}$ for $30 \mathrm{~min}$ eliminated the surface organic groups of Aerogel B, and therefore endowed the material with the highest surface area $\left(907 \mathrm{~m}^{2} / \mathrm{g}\right)$, pore size $(21.0 \mathrm{~nm})$ and total pore volume $\left(2.95 \mathrm{~m}^{3} / \mathrm{g}\right)$, and the smallest carbon and hydrogen contents $(0.89 \%$ and $0.23 \%)$. Xerogel $\mathrm{C}$, which did not undergo surface modification and therefore shrank greatly due to the capillary stress during the APD, showed a much smaller pore size and pore volume than those of Aerogel $A$ and $B$.

Fig. 3 shows the nitrogen adsorption-desorption isotherms and BJH pore size distribution of silica aerogels. From Fig. 3(a) we can see that both hydrophobic and hydrophilic silica aerogels exhibit type-IV adsorption isotherms, which indicate the presence of mesopores [17]. The desorption cycles of the isotherms
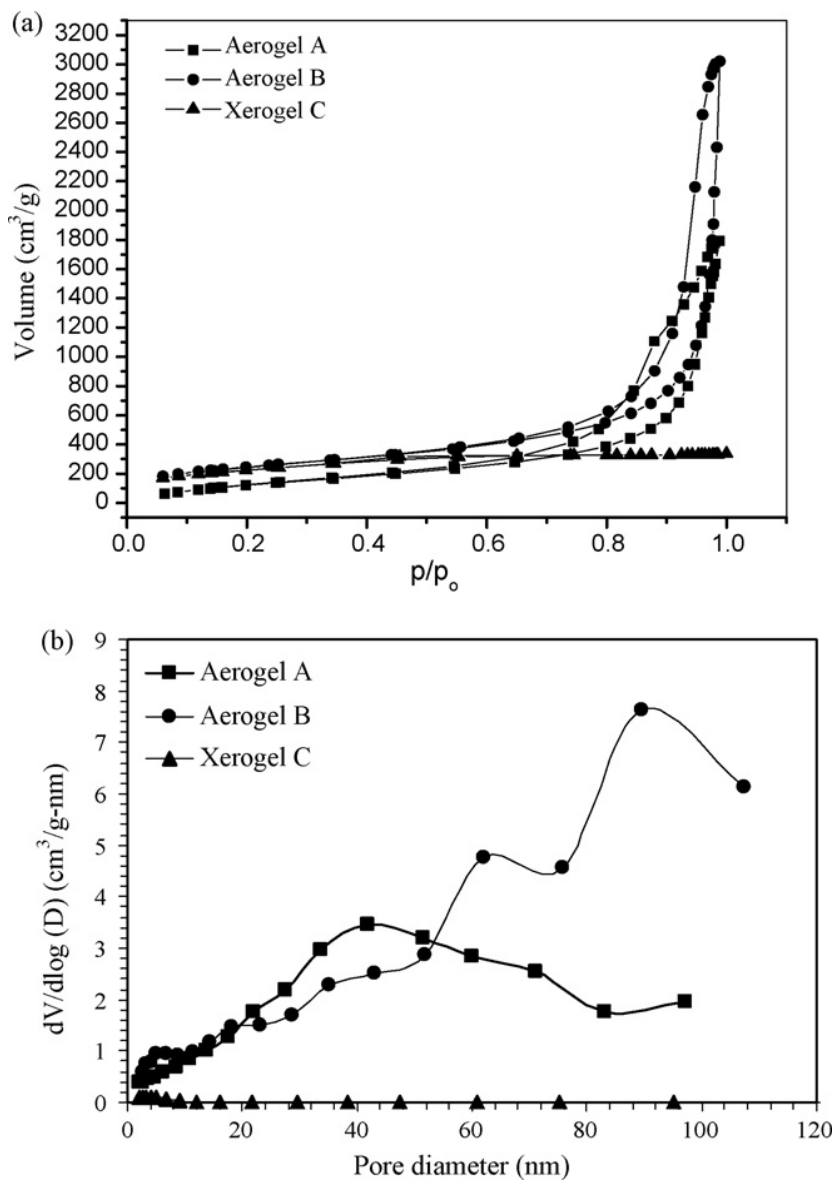

Fig. 3. $\mathrm{N}_{2}$ adsorption-desorption isotherms (a) and pore size distribution (b) of Aerogel A, Berogel B and Xerogel C. showed a hysteresis loop which is generally attributed to the capillary condensation occurring in the mesopores [18]. In addition, the adsorption isotherms are very similar to that of the supercritically dried aerogels [19]. It can be seen from Fig. 3(b) that Aerogel A contained pores below $60 \mathrm{~nm}$ with peak pore diameter residing at $40 \mathrm{~nm}$. With methyl groups of Aerogel A were calcined, the peak pore diameter of Aerogel B shifted to a higher side (ca. $90 \mathrm{~nm}$ ) with relatively broader pore size distribution.

\subsubsection{Infrared spectra of the aerogels and xerogel}

The FTIR investigations of the three materials are shown in Fig. 4. The peaks of $3433 \mathrm{~cm}^{-1}$ and $962 \mathrm{~cm}^{-1}$ for Xerogel C corresponded to the $\mathrm{O}-\mathrm{H}$ adsorption bond, which was caused by physically adsorbed water. The absorption peaks near $1092 \mathrm{~cm}^{-1}, 799 \mathrm{~cm}^{-1}$ and $468 \mathrm{~cm}^{-1}$ were due to $\mathrm{Si}-\mathrm{O}-\mathrm{Si}$ vibrations [14]. After the surface modification, Aerogel A showed visible absorption peaks at $2967 \mathrm{~cm}^{-1}, 1259 \mathrm{~cm}^{-1}$ and $847 \mathrm{~cm}^{-1}$, which correspond to $-\mathrm{CH}_{3}$ terminal groups. The adsorption peaks of the $\mathrm{O}-\mathrm{H}$ bond weakened as Aerogel A became hydrophobic with fewer adsorbed water molecules. In Aerogel B, which was calcined at $500^{\circ} \mathrm{C}$ for $30 \mathrm{~min}$ in the oven, the $-\mathrm{CH}_{3}$ adsorption peaks disappeared or greatly weakened as the methyl terminal groups were oxidized. The FTIR peak distribution of Aerogel B was much more like Aerogel C, which indicates that they had similar surface chemical properties.

\subsection{Thermal analysis of silica aerogels}

Fig. 5 illustrates DSC-TGA curve of Aerogel A that experienced surface modification by EtOH/TMCS/hexane. As it can be seen, there is a very little weight loss up to a temperature of $390^{\circ} \mathrm{C}$. A further increase of temperature causes weight loss along with an exothermic peak corresponding to the oxidation of the surface methyl group developed from the surface modification. This is the reason why aerogel samples become hydrophilic after heating at temperature of around $500^{\circ} \mathrm{C}$.

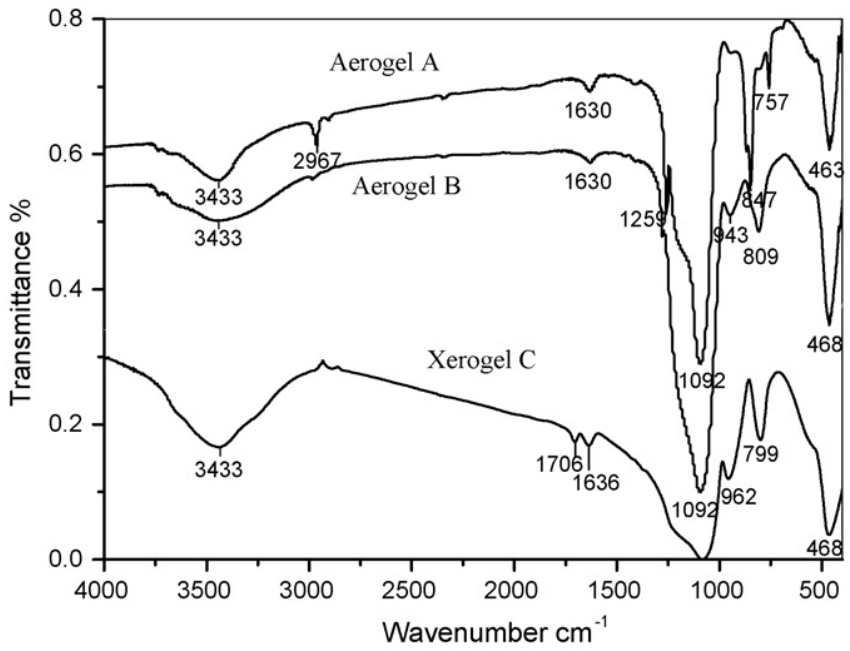

Fig. 4. Infrared spectra of Aerogel A, B and Xerogel C. 


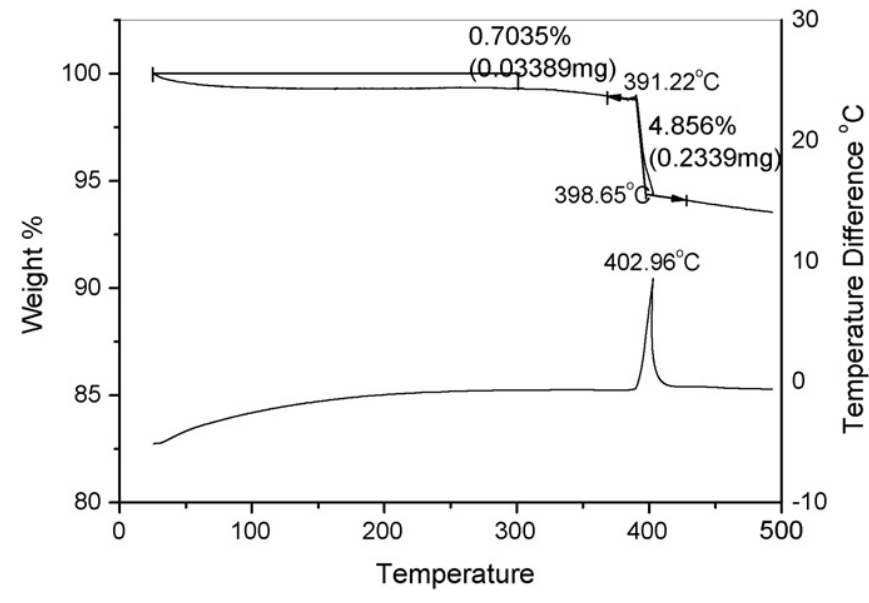

Fig. 5. DSC-TGA of Aerogel A.

\subsection{Adsorption capacity}

\subsubsection{Adsorption curves of RhB and dieldrin}

As shown in the adsorption curves of the three materials on RhB, Fig. 6(a), Aerogel B exhibits the best adsorption capacity by removing 95\% adsorbate within $1 \mathrm{~h}$. This removal capacity is simi-
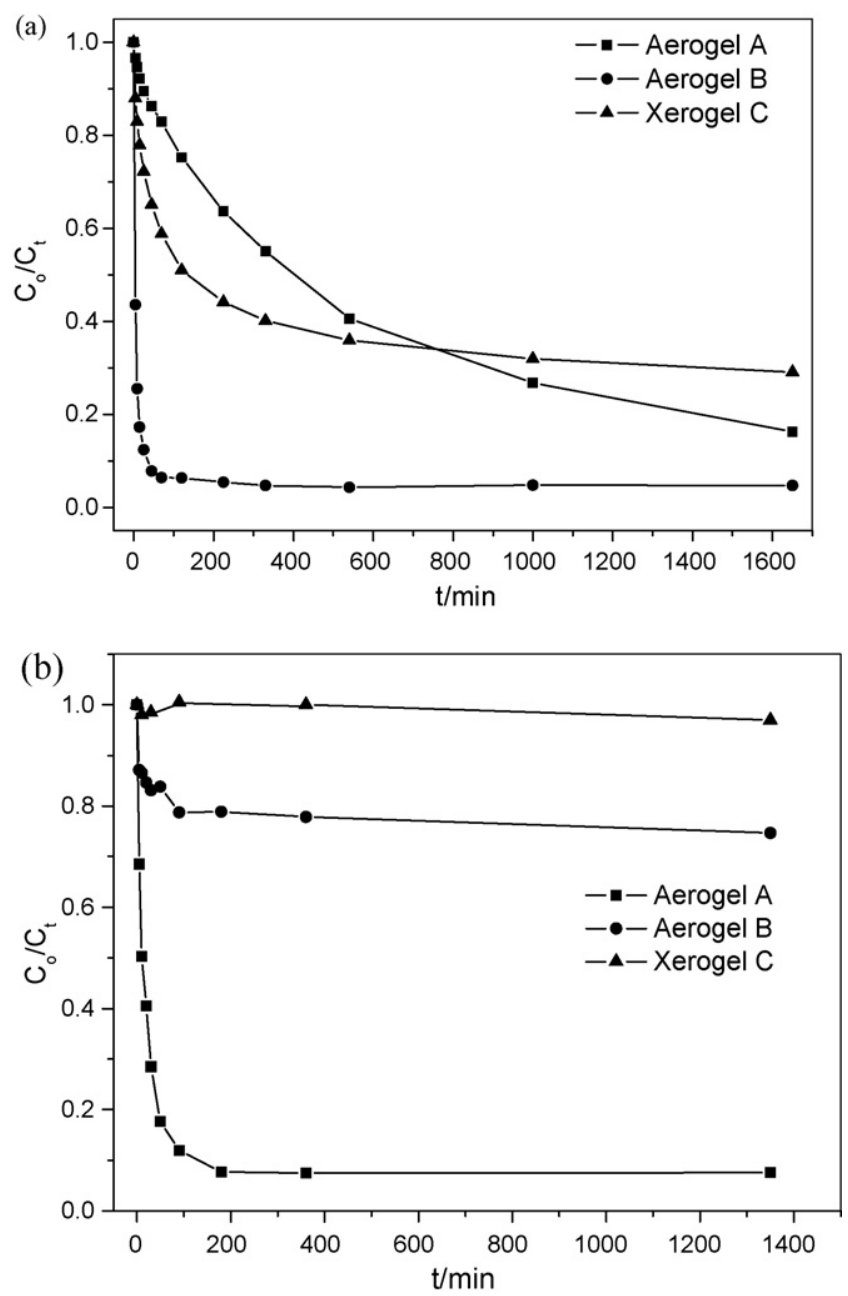

Fig. 6. Adsorption curve of RhB (a) and dieldrin (b) on Aerogel A, B and Xerogel C as a function of time. (a: $C_{0}=10 \mathrm{mg} / \mathrm{L} ; V=100 \mathrm{~mL} ; T=25^{\circ} \mathrm{C} ; N=170 \mathrm{rpm}$; adsorbent: $0.5 \mathrm{~g} ; \mathrm{b}: C_{0}=150 \mu \mathrm{g} / \mathrm{L} ; V=150 \mathrm{~mL} ; T=25^{\circ} \mathrm{C} ; N=170 \mathrm{rpm}$; adsorbent: $0.1 \mathrm{~g}$ ). lar to other popular catalytic degradation methods for RhB removal [20-21]. The highly porous structure and the affinity between the hydrophilic surface of Aerogel B and the RhB molecule were the primary reasons for the high adsorption effectiveness. Aerogel A, on the other hand, did not reach adsorption equilibrium even after $25 \mathrm{~h}$. This is likely due to the hydrophobic surface of Aerogel A, which repelled the aqueous phase and limited the through contact with adsorbate molecule. The medium adsorption velocity and limited capacity of Xerogel $C$ are attributable to its hydrophilic surface and compact microstructure.

Fig. 6(b) shows the differences of the three materials on the adsorption of dieldrin from water. Aerogel A shows strong adsorption effectiveness of the slightly soluble compound by removing 92.3\% dieldrin within $3 \mathrm{~h}$ when the system reached adsorption equilibrium. The hydrophobic surface of Aerogel A limited the competition of the water molecule to the adsorption sites, and ensured a strong affinity of the lipophilic dieldrin molecule. Although Aerogel B had even greater porosity than Aerogel A, it exhibited poor adsorption capacity on the same adsorbate by adsorbing only $21.5 \%$ dieldrin within $3 \mathrm{~h}$ at which point it reached saturation. The hydrophilic surface property that resulted from the calcination treatment rendered a stable bond between the adsorption sites and the water molecules, which negatively affected the adsorption effectiveness of Aerogel B for dieldrin. The limited affinity between the hydrophilic surface of Aerogel B and the lipophilic dieldrin molecule was another reason for the poor adsorption capacity. Xerogel $\mathrm{C}$ did not show any adsorption capacity for dieldrin.
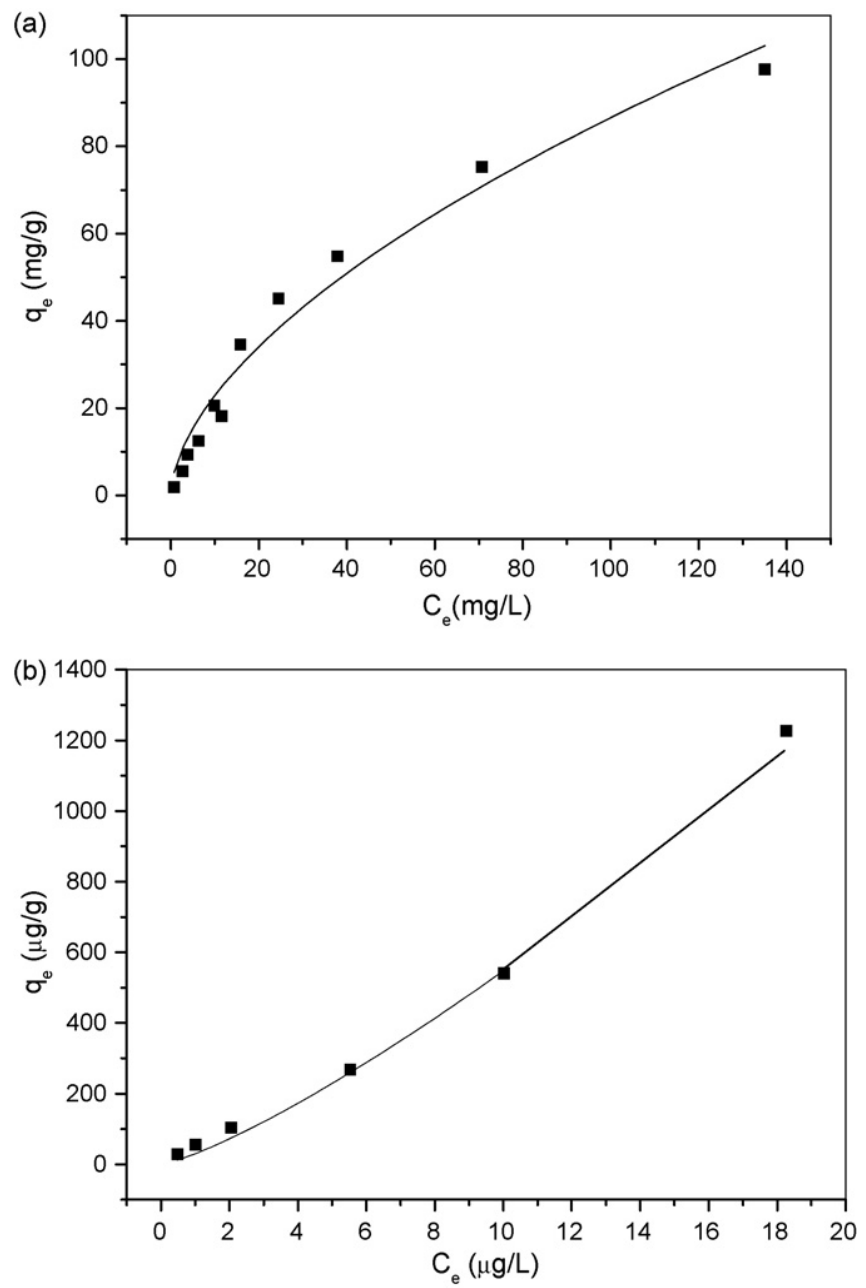

Fig. 7. Adsorption isotherm of RhB on hydrophilic silica aerogel (a) and dieldrin on hydrophobic silica aerogel (b). 


\subsubsection{Adsorption equilibrium}

The adsorption isotherms of RhB on Aerogel B and dieldrin on Aerogel A were presented in Fig. 7(a) and (b), respectively.

The correlation of equilibrium data using either a theoretical or empirical equation is essential for adsorption data interpretation and prediction. An important model widely used in removal of hydrophobic organic pollutants from water is the Freundlich isotherm representing a non-linear equilibrium between the phases (Eq. (8)).

$q_{\mathrm{e}}=K_{\mathrm{f}} C_{\mathrm{e}}^{1 / n}$

where $q_{\mathrm{e}}$ is the adsorption capacity of an adsorbent, $C_{\mathrm{e}}$ is the equilibrium concentration of the adsorbate, and $K_{\mathrm{f}}$ is an indication of the adsorption capacity of the adsorbent, while the parameter $n$ indicates the effect of concentration on the adsorption capacity and represents the adsorption intensity.The adsorption capacity of an adsorbent, $q_{\mathrm{e}}$, is calculated by means of equilibrium studies. Eq. (9) establishes the mass balance of a process at equilibrium condition:

$q_{\mathrm{e}}=\frac{V\left(C_{0}-C_{\mathrm{e}}\right)}{m}$

where $C_{0}$ is the initial concentration of the adsorbate, $m$ is the adsorbent mass and $V$ is the solution volume.

Results showed that aerogel presented unique adsorption patterns and could remove $\mathrm{RhB}$ and dieldrin from aqueous solution effectively. It can be seen from Fig. 7(a) that Aerogel B was more effective at lower RhB concentration. The $K_{\mathrm{f}}$ and $n$ of Aerogel B on
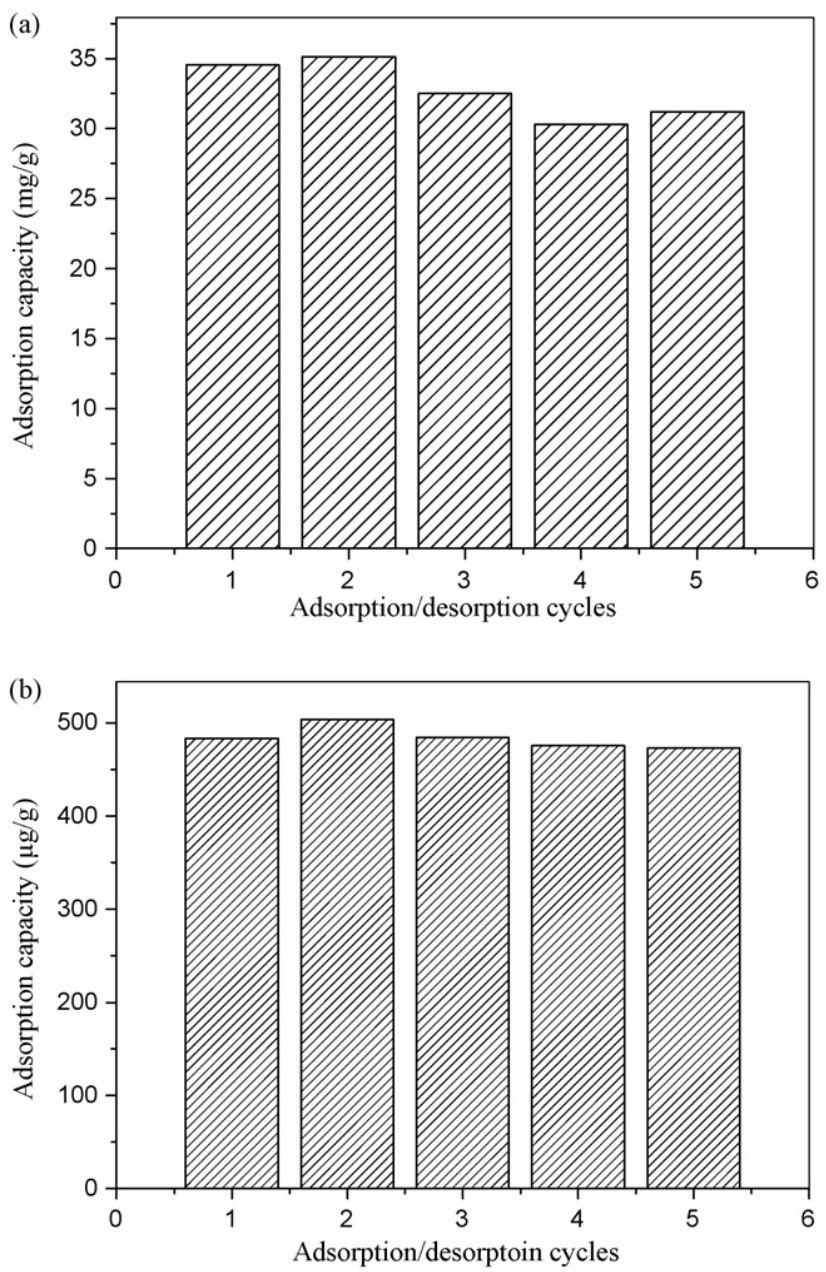

Fig. 8. Comparison of adsorption capacity of RhB on Aerogel B (a) and dieldrin on Aerogel A (b) after 5 adsorption/desorption cycles.
RhB were 1.16 and 0.73 , respectively. On the other hand, Aerogel A exhibited a very strong adsorption capacity on dieldrin, the $K_{\mathrm{f}}$ was 30.22, 11 times greater than that of active carbon, and 10 times greater than that of cellulose acetate [22]. Besides the high porosity and lipophilic surface, low density also played an important role for the high adsorption capacity exhibited by Aerogel A.

\subsubsection{Reusability of aerogel adsorbents}

To test the reusability of the aerogels, repeated adsorption/desorption cycles of Aerogel A on RhB and Aerogel B on dieldrin were performed. In the repeated adsorption/desorption experiments, Aerogel A and B were regenerated by thermal treatment at $300^{\circ} \mathrm{C}$ and $500{ }^{\circ} \mathrm{C}$, respectively, for $4 \mathrm{~h}$ to completely remove the adsorbed adsorbates. The adsorption steps were conducted after the complete desorption by thermal treatment. The adsorption capacity of both hydrophilic and hydrophobic aerogels were not influenced by desorption steps and remain stable at least 5 cycles, as shown in Fig. 8(a) and (b).

\section{Conclusions}

In this study silica aerogels were prepared with different hydrophobicity via a low-cost ambient pressure drying procedure. The synthesized materials had high surface area, pore diameter and pore volume. The physical and chemical properties of the silica aerogels were adjusted by surface modification and calcination.

Hydrophilic aerogel was effective in removing soluble organic matter from aqueous solution, while hydrophobic aerogel showed much stronger adsorption effectiveness on slightly soluble organic compounds. Aerogel adsorbents could be easily regenerated by calcination treatment and the adsorption capacities were not significantly affected. Due to the high adsorption efficiency on a wide range of organic compounds in water, easy modification of the properties, and the relative low manufacture cost, silica aerogel prepared via APD has great application potential in the water treatment field.

\section{References}

[1] K.C. Jones, P. de Voogt, Persistent organic pollutants (POPs): state of the science, Environ. Pollut. 100 (1999) 209-211.

[2] M. Toyoda, J. Aizawa, M. Inagaki, Sorption and recovery of heavy oil by using exfoliated graphite, Desalination 115 (1998) 199-201.

[3] L. Li, P.A. Quinlivan, D.R.U. Knappe, Effects of activated carbon surface chemistry and pore structure on adsorption of organic contaminants from aqueous solution, Carbon 40 (2002) 2085-2100.

[4] G.S. Kim, S.H. Hyun, Synthesis of window glazing coated with silica aerogel films via ambient drying. J. Non-Cryst. Solids 320 (2003) 125-132.

[5] W.C. Ackerman, M. Vlachos, S.R. Rouanet, J. Fruendt, Use of surface treated aerogels derived from various silica precursors in translucent insulation panels, J. Non-Cryst. Solids 285 (2001) 264-271.

[6] L.W. Hrubesh, Aerogel applications, J. Non-Cryst. Solids 225 (1998) 335-342.

[7] L.W. Hrubesh, P.R. Coronado, J.H. Satcher Jr., Solvent removal from water with hydrophobic aerogels, J. Non-Cryst. Solids 285 (2001) 328-332.

[8] S. Standeker, Z. Novak, Ž. Knez, Adsorption of toxic organic compounds from water with hydrophobic silica aerogels, J. Colloid Interface Sci. 310 (2007) 268-362.

[9] A.V. Rao, N.D. Hegde, H. Hirashima, Absorption and desorption of organic liquids in elastic superhydrophobic silica aerogels, J. Colloid Interface Sci. 305 (2007) $124-132$.

[10] A.V.Tao, R.R. Kalesh, Comparative studies of the physical and hydrophobic properties of TEOS based silica aerogels using different co-precursors, Sci. Technol. Adv. Mater. 4 (2003) 509-515.

[11] B. Unlusu, S.G. Sunol, A.K. Sunol, Stress formation during heating in supercritical drying, J. Non-Cryst. Solids 279 (2001) 110-118.

[12] C.J. Lee, G.S. Kim, S.H. Hyun, Synthesis of silica aerogels from waterglass via new modified ambient drying, J. Mater. Sci. 37 (2002) 2237-2241.

[13] S.D. Bhagat, Y.H. Kim, Y.S. Ahn, J.G. Yeo, Rapid synthesis of water-glass based aerogel by in situ surface modification of the hydrogels, Appl. Surf. Sci. 253 (2007) 3231-3236.

[14] F. Shi, L.J. Wang, J.X. Liu, Synthesis and characterization of silica aerogels by a novel fast ambient pressure drying process, Mater. Lett. 60 (2006) 3718-3722. 
[15] F. Schwertfeger, D. Frank, M. Schmidt, Hydrophobic waterglass based aerogels without solvent exchange or supercritical drying, J. Non-Cryst. Solids 225 (1998) 24-29.

[16] W. Sha, R.P. Liu, H.J. Liu, J.H. Qu, Characterization of silica aerogel prepared by ambient drying and it's adsorption capacity for dieldrin, Environ. Sci., in press, in Chinese with English abstract.

[17] J. Haber, A. Baiker, M. Nobbenhuis, J. Kiwi, R. Thampi, M. Gratzel, Surface-area and porosity, Catal. Today 20 (1994) 11-16.

[18] S.D. Bhagat, Y.H. Kim, M.J. Moon, Y.S. Ahn, J.G. Yeo, A cost-effective and fast synthesis of nanoporous $\mathrm{SiO}_{2}$ aerogel powders using water-glass via ambient pressure drying route, Solid State Sci. 9 (2007) 628-635.
[19] J.H. Lee, S.Y. Choi, C.E. Kim, G.D. Kim, The effects of initial sol parameters on the microstructure and optical transparency of $\mathrm{TiO}_{2}-\mathrm{SiO}_{2}$ binary aerogels, J. Mater. Sci. 32 (1997) 3577-3585.

[20] J.Q. Li, L.P. Li, L. Zheng, Y.Z. Xian, L.T. Jin, Photoelectrocatalytical degradation of rhodamine $\mathrm{B}$ using $\mathrm{Ti} / \mathrm{TiO}_{2}$ electrode prepared by laser calcination method, Electrochim. Acta 51 (2006) 4942-4949.

[21] P. Wilhelm, D. Stephan, Photodegradation of rhodamine B in aqueous solution via $\mathrm{SiO}_{2} @ \mathrm{TiO}_{2}$ nano-spheres, J. Photochem. Photobiol. A 185 (2007) 19-25.

[22] J. Ru, H.J. Liu, J.H. Qu, A.M. Wang, R.H. Dai, Removal of dieldrin from aqueous solution by a novel triolein-embedded composite adsorbent, J. Hazard. Mater. 141 (2007) 61-69. 